\title{
Divergência genética entre progênies de polinização aberta de Eucalyptus tereticornis Smith para caracteres de crescimento e de qualidade da madeira
}

\author{
Genetic divergence among Eucalyptus tereticornis Smith open pollinated \\ progenies for growth and wood quality characters
}

\author{
Bruna Zanatto $^{1}$ (D), Rinaldo Cesar de Paula ${ }^{1}$ (D), Nádia Figueiredo de Paula² (D), \\ Miguel Luiz Menezes Freitas ${ }^{3}$ (D), Marcio José de Araújo ${ }^{1}$ (D) \\ ${ }^{1}$ Universidade Estadual Paulista - Unesp, Faculdade de Ciências Agrárias e Veterinárias, Jaboticabal - FCAV, Jaboticabal, SP, \\ Brasil \\ ${ }^{2}$ Faculdade de Tecnologia de Jaboticabal "Nilo de Stéfani"- FATEC Jaboticabal, Jaboticabal, SP, Brasil \\ ${ }^{3}$ Instituto Florestal de São Paulo - IF, Secretaria do Meio Ambiente do Estado de São Paulo, São Paulo, SP, Brasil
}

\begin{abstract}
Como citar: Zanatto, B., Paula, R. C., Paula, N. F., Freitas, M. L. M., \& Araújo, M. J. (2020). Divergência genética entre progênies de polinização aberta de Eucalyptus tereticornis Smith para caracteres de crescimento e de qualidade da madeira. Scientia Forestalis, 48(128), e3327. https://doi.org/10.18671/scifor.v48n128.15
\end{abstract}

\begin{abstract}
Resumo
O Eucalyptus tereticornis Smith tem boa adaptabilidade a diferentes regiões e resistência à seca, apresentando crescimento rápido e grande potencial de aplicação da madeira para diferentes usos. No Brasil, há poucos estudos com a espécie, necessitando-se avaliar a divergência genética entre os acessos disponíveis em testes de procedências e progênies espalhados pelo país. Neste sentido, o presente projeto visou avaliar a divergência genética entre progênies de polinização aberta de $E$. tereticornis com base em caracteres de crescimento e de qualidadeda madeira. O experimento estudado foi implantado no município de Luiz Antônio (SP), com 30 progênies de E. tereticornis, pertencentes a duas procedências. Com 31 anos de idade, foram avaliados o diâmetro à altura do peito, altura total, volume com casca, biomassa do tronco, densidade básica (DBM), teores de celulose (CEL), lignina (LIG), extrativos (EXT) e holocelulose (HOLO) na madeira. Foram amostradas três árvores representativas de cada progênie para determinação do volume e obtenção de discos em diferentes posições do fuste para a determinação da DBM e dos caracteres de qualidade da madeira. Procedeu-se a análise multivariada, pelo método hierárquico de agrupamento de Ward e não hierárquico de K-means (K-médias) e a análise de componentes principais a partir da matriz de correlação. Pelo método de Ward as progênies foram divididas em três grupos, sendo que os grupos 1 e 2, de maiores produtividades, diferenciaram pelos caracteres de qualidade da madeira e o grupo 3 reuniu progênies de menor produtividade. No método de K-means, os três grupos formados diferenciaram-se pouco daqueles obtidos no método de Ward, porém apresentaram características mais bem definidas. Na análise por componentes principais os três primeiros componentes explicaram $78,76 \%$ da variância contida nas variáveis originais. De maneira geral os caracteres de qualidade da madeira apresentaram uma quase-independência do potencial de crescimento das árvores. Os caracteres em destaque nos três componentes principais têm grande importância para seleção das progênies mais promissoras para um programa de melhoramento. $\mathrm{O}$ componente principal 1 (CP1), está relacionado com a produtividade das progênies, o CP2 com LIG e HOLO e o CP3 com DBM e CEL. A presença de divergência genética entre as 30 progênies de polinização aberta de Eucalyptus tereticornis não está relacionada à procedência das sementes. As progênies avaliadas diferem quanto ao potencial produtivo e caracteres de qualidadeda madeira. Os grupos identificados podem ser utilizados nas estratégias de melhoramento de modo a selecionar progênies
\end{abstract}

Fonte de financiamento: Coordenação de Aperfeiçoamento de Pessoal de Nível Superior (CAPES) - Código de Financiamento 001.

Conflito de interesse: Nada a declarar.

Autor correspondente: rinaldo.paula@unesp.br

Recebido: 2 abril 2019.

Aceito: 6 novembro 2019

Editor: Paulo Henrique Müller Silva.

(c) (i) Este é um artigo publicado em acesso aberto (Open Access) sob a licença Creative Commons Attribution, que permite uso, distribuição e (c) reprodução em qualquer meio, sem restrições desde que o trabalho original seja corretamente citado. 
superiores com vistas à obtenção de ganho genético em produtividade e melhoria da qualidade da madeira para segmentos distintos, de acordo com o objetivo do programa de melhoramento.

Palavras-chave: Qualidade da madeira; Melhoramento florestal; Análise multivariada.

\begin{abstract}
Eucalyptus tereticornis Smith has good adaptability to different regions and drought resistance, presenting fast growth and great potential of wood for different uses. In Brazil, there are few studies with this species, needing to evaluate the genetic divergence between the accessions available in provenances and progeny tests spread throughout the country. In this sense, the present work aimed to evaluate the genetic divergence between open-pollinated progenies of E. tereticornis based on wood growth and chemical traits. The experiment was carried out near the town of Luiz Antônio (SP), with 30 progenies of E. tereticornis, belonging to two origins. At 31 years of age, diameter at breast height, total height, commercial volume with bark, trunk biomass, basic density (DBM), cellulose (CEL), lignin (LIG), extractives (EXT) and holocellulose (HOLO) contents in wood. Three representative trees of each progeny were selected to determine the volume and obtain discs in different stem positions to determine the chemical wood traits. Multivariate analysis was performed by the Ward hierarchical method and non-hierarchical K-means (K-means) and the principal component analysis from the correlation matrix. By Ward's method, the progenies were divided into three groups, and groups 1 and 2, with higher yields, differed by the chemical wood traits from group 3, which presented a lower productivity. By the K-means method, the three groups differed little from those obtained by the Ward method, but had better defined traits. In the principal component analysis the first three components explained $78.76 \%$ of the variance contained in the original variables. In general, the chemical wood traits showed a quasi-independence of the tree growth potential. The highlighted characters in the three main components are of great importance for selecting the most promising progenies for a breeding program. The main component 1 (CP1) is related to the progeny productivity, CP2 with LIG and HOLO and CP3 with DBM and CEL. The presence of genetic divergence among the 30 open pollinated progenies of Eucalyptus tereticornis is not related to seed origin. The progenies evaluated differed in the productive potential and quality of wood traits. The identified groups can be used in breeding strategies in order to select superior progenies for genetic gain in productivity and wood quality improvement for different segments, according to the breeding program objective.
\end{abstract}

Keywords: Wood quality; Forest improvement; Multivariate analysis.

\title{
INTRODUÇÃO
}

O setor brasileiro de florestas plantadas ocupa uma área de 7,83 milhões de hectares (Indústria Brasileira de Árvores, 2019), sendo responsável por $91 \%$ de toda a madeira produzida para fins industriais no país. Entre os segmentos que utilizam a madeira como principal matéria-prima, estão os setores produtivos de celulose e papel, painéis de madeira, pisos laminados, serrados e compensados, siderurgia a carvão vegetal e de energia. No setor de bens de consumo, destacam-se as indústrias gráficas, de embalagem, moveleira, química, têxtil, farmacêutica e a construção civil (Indústria Brasileira de Árvores, 2017).

O cultivo do eucalipto tem expandido para áreas com limitações hídricas e nutricionais e, mesmo em regiões onde a silvicultura já está bem estabelecida, irregularidades climáticas recentes, a exemplo do extenso período de estiagem ocorrido entre 2014 e 2016 no estado de Minas Gerais, causaram a perda de cerca de 200 mil ha de florestas de eucalipto (Gonçalves et al., 2017). Não obstante a isto, novas demandas para a produção de energia de biomassa aumentam a procura por espécies de eucalipto para esta finalidade. Neste sentido, Eucalyptus tereticornis Smith é uma espécie que atende, simultaneamente, a estas duas situações atuais, pois é considerada tolerante à seca (Flores et al., 2016) e com madeira de boa qualidade para a produção de energia, produtos sólidos, dentre outros (Instituto de Pesquisas e Estudos Florestais, 2018).

Eucalyptus tereticornis inclui as subespécies tereticornis e mediana (Brooker \& Kleinig, 2006; Fonseca et al., 2010), cuja área de ocorrência natural compreende os Estados de Queensland, New South Wales, Victoria atingindo até Papua Nova Guiné, em faixa de latitude de 6 a $38^{\circ} \mathrm{S}$, altitudes que podem variar desde o nível do mar até $2.500 \mathrm{~m}$, com precipitação anual de 600 a $3.000 \mathrm{~mm}$, temperaturas médias entre 8 e $27^{\circ} \mathrm{C}$ (Flores et al., 2016), e temperatura média das máximas entre 22 e $32^{\circ} \mathrm{C}$ (Balbinot et al., 2010). Desenvolve-se melhor em solos profundos, bem drenados, neutros ou ligeiramente ácidos, vegetando muito bem 
no período seco e pode suportar pequenos alagamentos (Silva et al., 2007). E. tereticornis tem ampla adaptação ambiental quanto ao estresse hídrico, distribuindo-se desde as regiões semiáridas até as muito úmidas (Flores et al., 2016).

Dentre as características da madeira, a densidade básica é a mais utilizada em programas de melhoramento florestal e na seleção de árvores, por ser de fácil determinação, apresentar boa correlação com as propriedades físicas e mecânicas da madeira e composição celular e, também, pela alta herdabilidade encontrada (Fernandes et al., 2011; Vital et al., 2013). Contudo, Foelkel et al. (1992) alertam sobre o uso isolado da densidade básica para definir a qualidade da madeira e afirmam que a densidade é um fator resultante, ou seja, é a consequência e não a causa da distribuição, tipos e composição dos elementos anatômicos que compõem a madeira.

Para a avaliação da qualidade da madeira para um determinado uso, além da densidade básica, outras características devem ser consideradas, a exemplo dos principais constituintes químicos como os teores de celulose, hemicelulose, lignina e de extrativos que representam, em média, na madeira das principais espécies de eucalipto cultivadas no Brasil, 45\%, de 20 a $25 \%$, de 25 a 30\% e menos que 3\%, respectivamente (Gomide \& Colodette, 2007; Morais et al., 2005; Rowell et al., 2005; Trugilho et al., 1996; Vital et al., 2013).

$\mathrm{Na}$ caracterização de acessos para estudos de potencial de melhoramento e, ou conservação genética, em geral, vários caracteres são avaliados e, nesse contexto, as técnicas multivariadas têm sido amplamente usadas e com sucesso para medir a diversidade genética entre os genótipos (Alves et al., 2003), permitindo, por exemplo, o direcionamento de cruzamentos em programas de hibridação e a eliminação de acessos redundantes em bancos de germoplasma.

O Instituto Florestal de São Paulo implantou, na década de 1980, testes de procedências e progênies de Eucalyptus tereticornis. Pouco, ainda, se conhece sobre a base genética desta espécie disponível aqui no Brasil, de forma que a avaliação desses testes assume grande importância, pois poderão disponibilizar informações sobre a variabilidade genética das populações estabelecidas no Brasil que possam atender as novas demandas do setor florestal brasileiro. Neste sentido, o presente trabalho objetivou avaliar a divergência genética entre progênies de polinização aberta de Eucalyptus tereticornis a partir de caracteres de crescimento e de qualidade da madeira.

\section{MATERIAL E MÉTODOS}

Foram usados dados de um teste de progênies de polinização aberta de Eucalyptus tereticornis Smith, com 31 anos de idade, constituído por 30 progênies de polinização aberta, pertencentes a duas procedências: Ravenshoe (Progênies P153 a P170) e Mt. Garnet (Progênies P171 a P182), ambas da Austrália. O teste foi implantado em 11 de novembro de 1985 na Estação Experimental de Luiz Antônio, pertencente ao Instituto Florestal de São Paulo, no município de Luiz Antonio-SP. A precipitação média histórica no município de Luiz Antonio é de $1.429 \mathrm{~mm}$, com temperatura média anual de $21,2^{\circ} \mathrm{C}$, com variação entre 17,6 a $23,5^{\circ} \mathrm{C}$, temperaturas mínimas entre 10,7 a $18,1^{\circ} \mathrm{C}$ e temperaturas máximas entre 25,5 a $29,1^{\circ} \mathrm{C}$ (Climate-Date, 2018). Usou-se o delineamento experimental de blocos completos casualizados, com 100 repetições de uma planta, no espaçamento $4 \times 4$ m, sem adubação e sem bordadura, em um solo classificado como Latossolo Vermelho (Santos et al., 2013).

Após a avaliação do diâmetro à altura do peito (DAP), com suta graduada em $\mathrm{mm}$, da altura total (ALTT, m), com um Relascópio de Bitterlich, e da sobrevivência (SOB, \%) obtida a partir do levantamento das árvores vivas em todo o teste, foram amostradas e abatidas três árvores de cada progênie, totalizando 90 árvores. Destas árvores foram retirados seis discos de aproximadamente $5 \mathrm{~cm}$ de espessura, correspondentes a 0\%, DAP, $25 \%, 50 \%, 75 \%$ e $100 \%$ do comprimento do fuste (altura comercial, até o diâmetro mínimo de $4 \mathrm{~cm}$ ). Nestes discos foram determinados os diâmetros médios com e sem casca, a partir de duas avaliações perpendiculares. De posse do comprimento do fuste e dos diâmetros avaliados ao longo do 
mesmo, foram calculados os volumes com (VCC, $\mathrm{m}^{3}$ árvore-1) e sem casca, empregando-se a fórmula de Smalian, conforme citado por Soares et al. (2006).

Os discos de madeira devidamente identificados, foram levados para o laboratório para secagem. De cada disco foram retiradas duas amostras, em forma de cunha, sendo uma destinada para determinação da densidade da madeira e casca e outra para análises químicas. As cunhas destinadas à análise química foram picadas e, posteriormente, moídas em moinho tipo Willey.

Densidade básica da madeira e da casca - foram determinadas pelo método da imersão em água, de acordo com Vital (1984), nas cunhas e, ou casca obtidas ao longo do fuste, correspondentes aos pontos de amostragem dos discos.

Biomassa do tronco (BIOMT, kg árvore ${ }^{-1}$ ) - obtida pela soma do produto entre densidade da madeira e da casca e os respectivos volumes.

As determinações químicas foram realizadas a partir de uma amostra composta de madeira moída, retirada dos diferentes discos. Foram determinados os teores de extrativos, de lignina, de celulose e holocelulose:

Eliminação de Extrativos- antes da determinação dos teores de lignina e celulose foi realizada a eliminação dos extrativos, usando $5 \mathrm{~g}$ de amostra seca de madeira de cada progênie, realizando a extração em água e em solução com tolueno e álcool etílico (1:2 v/v).

Teor de Lignina (LIG, \%) - após eliminação dos extrativos, o teor de lignina foi determinado pelo método Klason, modificado de acordo com o procedimento proposto por Gomide \& Demuner (1986), usando-se amostras de 0,3 g de madeira moída

Teor de Celulose Total (CEL, \%) - foi determinado pelo método do ácido nítrico, conforme procedimento descrito em Santos \& Sansígolo (2000).

Teor de Holocelulose (HOLO, \%) - obtido indiretamente, por diferença em relação ao total (100\%), conforme a expressão: HOLO = 100 - LIG - EXT - CEL .

Teor de Extrativos (EXT, \%) - obtido com uma amostra de madeira moída, a qual foi colocada em cartuchos de papel, pesadas e levadas a um digestor com Diclorometano (solvente), pingando sobre os cartuchos de papel por 8 horas assim eliminando os extrativos da madeira moída. Posteriormente foram levados a estufa a $105^{\circ} \mathrm{C}$ por $12 \mathrm{~h}$, os cartuchos foram retirados da estufa, esfriados em dessecador e pesados. $O$ teor de extrativo foi obtido pela diferença de massa entre cartucho de papel com a amostra de madeira seca antes e após a extração.

Análise de Dados: os dados coletados (para os caracteres DAP, ALTT, VCC, BIOMT, DBM, LIG, CEL, HOLO e EXT) foram analisados sob modelos mistos com o uso do software Selegen REML/BLUP (Resende, 2007) segundo a seguinte equação:

$\mathbf{Y}=\mathbf{X r}+\mathbf{Z g}+\mathbf{W b}+\mathbf{e}$

em que y é o vetor dos dados; $r$ é o vetor dos efeitos de repetição (assumidos como fixos) somados à média geral; g é o vetor dos efeitos genotípicos (assumidos como aleatórios); b é o vetor dos efeitos de procedências (assumidos como aleatórios); e é o vetor de resíduos (aleatórios). Para o cálculo da herdabilidade no sentido restrito foi utilizado um coeficiente de parentesco $(r)$ de 0,4, sendo este mais apropriado para populações de polinização aberta de Eucalyptus (Eldridge et al., 1993; Williams et al., 2002).

Os valores genotípicos das famílias obtidos na análise descrita acima foram submetidos às análises multivariadas de agrupamento, pelo método hierárquico de Ward e não hierárquico K-means (K-médias) e de dispersão por análise de componentes principais (ACP), utilizando o Software STATISTICA 7.0 (StatSoft, 2004). Na análise pelo método hierárquico de Ward, a definição dos grupos foi obtida pelo método de Mojena (Mojena, 1977).

O número de grupos no método de K-means foi pré-definido a partir do número de grupos identificados no agrupamento de Ward. Na ACP o número de componentes principais foi definido de forma a explicar pelo menos $70 \%$ da variabilidade contida nos dados. 


\section{RESULTADOS E DISCUSSÃO}

As estimativas dos coeficientes de determinação do efeito de procedências ( $c_{\text {proc }}^{2}$ ), foram baixas para todas as características, denotando ser pequeno o efeito da procedência na variabilidade observada para os caracteres avaliados (Tabela 1). De maneira similar, as estimativas de herdabilidade no sentido restrito $\left(\mathrm{h}_{\mathrm{a}}^{2}\right)$ foram de baixas magnitudes para os caracteres de crescimento (DAP, VCC e BIOMT), e de moderada magnitude para ALTT (Tabela 1). Isso indica um baixo controle genético aditivo no crescimento das árvores, como é evidenciado em espécies florestais (Cornelius, 1994). Outros fatores que podem estar contribuindo para esses resultados são a idade avançada das árvores (31 anos) e o efeito de amostragem, pois a $\mathrm{h}_{\mathrm{a}}^{2}$ tende a diminuir com a idade (Volker et al., 2008; He et al., 2012) e amostras de pequeno tamanho (Resende et al., 1998).

As estimativas de $h_{a}^{2}$ para os caracteres de qualidade da madeira foram de média $(0,30$ para LIG) a alta (0,83 para CEL) magnitude, evidenciando maior controle genético aditivo em relação ao crescimento das árvores. Além disso, as herdabilidades a nível de média de progênies seguiram a mesma tendência, o que indica maior confiabilidade de selecionar progênies superiores para os caracteres de qualidade da madeira e baixo potencial de seleção para os caracteres de crescimento. Isso pode ser visualizado efetivamente comparando as acurácias entre as duas naturezas desses caracteres (Tabela 1). As condições climáticas no local do teste, são semelhantes às condições naturais das duas procendências, com grande similaridade quanto a pluviosidade de Ravenshoe e as temperaturas de Mt. Garnet. Desta forma, o pequeno efeito de procedências e o pequeno número de grupos formados (conforme será descrito à frente) podem ser função da idade de avaliação do teste, em que após 31 anos, a pressão exercida pelo ambiente tenha favorecido a sobrevivência (74\%) apenas dos indivíduos mais adaptados ao local de estudo. Isso pode ter resultado em comportamento relativamente semelhante dos indivíduos entre as progênies e procedências, principalmente em relação ao crescimento das árvores amostradas.

Tabela 1. Estimativas dos parâmetros genéticos para os caracteres de crescimento e de qualidade da madeira em progênies de polinização aberta de Eucalyptus tereticornis aos 31 anos de idade.

\begin{tabular}{cccccccccc}
\hline & DAP & ALTT & VCC & DBM & BIOMT & LIG & CEL & EXT & HOLO \\
\hline$\sigma_{a}^{2}$ & 0,143 & 2,878 & 0,0003 & 0,001 & 0,0003 & 2,326 & 5,050 & 0,959 & 4,605 \\
$\sigma_{\text {proc }}^{2}$ & 0,708 & 0,009 & 0,0003 & 0,000 & 0,0003 & 0,006 & 0,841 & 0,062 & 0,008 \\
$\sigma_{e}^{2}$ & 7,377 & 6,655 & 0,0170 & 0,002 & 0,0090 & 6,761 & 3,229 & 1,025 & 7,395 \\
$\sigma_{f}^{2}$ & 8,142 & 7,814 & 0,0170 & 0,002 & 0,0090 & 7,697 & 6,089 & 1,470 & 9,245 \\
$\boldsymbol{h}_{a}^{2}$ & 0,018 & 0,368 & 0,0140 & 0,375 & 0,0270 & 0,302 & 0,829 & 0,652 & 0,498 \\
$\boldsymbol{c}_{\text {proc }}^{2}$ & 0,087 & 0,001 & 0,0170 & 0,005 & 0,0330 & 0,001 & 0,138 & 0,042 & 0,001 \\
$\boldsymbol{h}_{\boldsymbol{m} p}^{2}$ & 0,023 & 0,342 & 0,025 & 0,343 & 0,024 & 0,292 & 0,652 & 0,529 & 0,428 \\
$\boldsymbol{A} \boldsymbol{c}_{\text {prog }}$ & 0,151 & 0,585 & 0,158 & 0,586 & 0,156 & 0,541 & 0,808 & 0,727 & 0,654 \\
CVe\% & 12,066 & 9,378 & 28,698 & 5,701 & 31,175 & 8,324 & 3,923 & 11,73 & 4,524 \\
Média Geral & 22,51 & 27,506 & 0,454 & 0,719 & 0,303 & 31,236 & 45,801 & 8,63 & 60,109 \\
\hline
\end{tabular}

$\sigma_{a}^{2}$ : variância genética aditiva; $\sigma_{p r o c}^{2}$ : variância entre procedências; $\sigma_{e}^{2}$ : variância residual; $\sigma_{f}^{2}$ : variância fenotípica individual; $\boldsymbol{h}_{a}^{2}$ : herdabilidade no sentido restrito; $c_{p r o c}^{2}$ : coeficiente de determinação dos efeitos de procedência; $\boldsymbol{h}_{\boldsymbol{m} p}^{2}$ : herdabilidade ajustada da média de genótipo, assumindo sobrevivência completa; $\boldsymbol{A} \boldsymbol{c}_{\boldsymbol{p r o g}}$ : acurácia da seleção de progênies e genitores; CVe\%: coeficiente de variação residual; DAP - Diâmetro à Altura do Peito (cm), ALTT - altura total $(\mathrm{m})$; VCC - volume comercial com casca $\left(\mathrm{m}^{3}\right.$ árvore-1); DBM - densidade básica da madeira $\left(\mathrm{g} \mathrm{cm}^{-3}\right)$, BIOMT - biomassa do tronco ( $\mathrm{kg}^{\text {rrvore }}{ }^{-1}$ ), LIG, CEL, EXT e HOLO - respectivamente, teores (\%) de lignina, celulose, extrativos e holocelulose.

As estimativas dos coeficientes de variação experimental (CVe\%) obtidos para os caracteres avaliados (Tabela 1), variaram de 3,92 para celulose (CEL) a 31,17\% para biomassa 
total (BIOMT). Os valores de CVe\% para espécies florestais, em condições de campo, mesmo em valores superiores aos reportados para outras culturas (Pimentel Gomes, 1990), revelam boa precisão experimental (Garcia, 1989).

Os resultados obtidos para os caracteres de qualidade da madeira (DBM, LIG, CEL, HOLO e EXT), são distintos daqueles comumente encontrados na literatura, em geral, obtidos de árvores com idades mais jovens. Assim, os valores médios aqui observados (Tabelas 2 e 3 ) de EXT (8,63\%), LIG (31,24\%), CEL $(45,80 \%)$ e HOLO $(60,11 \%)$ podem, em parte serem função da idade mais avançada do teste de progênies em estudo, o que corrobora com os resultados obtidos por Silva et al. (2005). Esses autores, estudando a composição química de Eucalyptus grandis com idades entre 10 e 25 anos, observaram tendência de aumento com a idade nos teores de extrativos totais, variando de 2,76\% (10 anos) a 6,34\% (20 anos) e de lignina total, passando de $23,78 \%$, para 30,70\% aos 10 e 20 anos, respectivamente. Silva et al. (2005) e Soares et al. (2015) confirmaram, em seus trabalhos, um aumento sistemático do teor de lignina total da madeira com a idade, porém com tendência de estabilização, após certa idade da árvore. O teor de holocelulose, por outro lado, apresentou tendência de redução com a idade, variando de $73,11 \%$ (14 anos) a 63,21\% (20 anos). O teste aqui avaliado apresenta idade superior ao estudado por Silva et al. (2005) e, portanto, os maiores teores observados de EXT e LIG e menores de HOLO, podem ser explicados pela idade maior das árvores e por tratar-se de uma outra espécie.

Tabela 2. Valores genotípicos preditos dos caracteres de crescimento e de qualidade da madeira em 30 progênies de polinização aberta de Eucalyptus tereticornis pertencentes a duas procedências Australianas.

\begin{tabular}{|c|c|c|c|c|c|c|c|c|c|c|}
\hline Procedência & Progênie & DAP & ALTT & VCC & DBM & BIOMT & LIG & CEL & EXT & HOLO \\
\hline \multirow[t]{18}{*}{ Ravenshoe } & 153 & 22,46 & 26,84 & 0,452 & 0,718 & 0,302 & 30,53 & 46,10 & 9,051 & 60,76 \\
\hline & 154 & 22,49 & 27,93 & 0,456 & 0,717 & 0,304 & 31,06 & 45,91 & 9,072 & 59,97 \\
\hline & 155 & 22,54 & 27,62 & 0,455 & 0,723 & 0,304 & 31,84 & 45,19 & 8,950 & 58,93 \\
\hline & 156 & 22,49 & 27,39 & 0,454 & 0,728 & 0,303 & 31,31 & 47,40 & 7,921 & 60,53 \\
\hline & 157 & 22,51 & 27,76 & 0,455 & 0,730 & 0,304 & 30,97 & 48,00 & 7,882 & 61,06 \\
\hline & 158 & 22,44 & 26,84 & 0,451 & 0,727 & 0,301 & 30,85 & 47,28 & 8,964 & 60,36 \\
\hline & 159 & 22,53 & 29,12 & 0,458 & 0,733 & 0,306 & 31,15 & 46,71 & 7,801 & 60,87 \\
\hline & 160 & 22,55 & 28,81 & 0,458 & 0,727 & 0,306 & 31,34 & 47,52 & 8,617 & 59,92 \\
\hline & 161 & 22,58 & 27,99 & 0,455 & 0,711 & 0,304 & 30,47 & 47,90 & 8,603 & 61,21 \\
\hline & 162 & 22,50 & 27,43 & 0,453 & 0,707 & 0,303 & 31,09 & 45,38 & 9,066 & 59,93 \\
\hline & 163 & 22,53 & 27,82 & 0,455 & 0,711 & 0,304 & 31,56 & 45,46 & 9,449 & 58,94 \\
\hline & 164 & 22,55 & 27,19 & 0,455 & 0,714 & 0,303 & 31,90 & 44,81 & 8,851 & 58,92 \\
\hline & 165 & 22,49 & 27,40 & 0,453 & 0,722 & 0,303 & 31,11 & 44,48 & 8,650 & 60,24 \\
\hline & 166 & 22,47 & 26,98 & 0,452 & 0,729 & 0,302 & 31,30 & 44,45 & 8,532 & 60,04 \\
\hline & 167 & 22,51 & 26,98 & 0,454 & 0,719 & 0,303 & 30,70 & 44,32 & 8,418 & 61,03 \\
\hline & 168 & 22,52 & 27,21 & 0,454 & 0,718 & 0,303 & 32,29 & 46,28 & 8,490 & 58,64 \\
\hline & 169 & 22,51 & 27,50 & 0,454 & 0,718 & 0,303 & 31,74 & 44,36 & 8,747 & 59,24 \\
\hline & 170 & 22,55 & 27,46 & 0,456 & 0,706 & 0,304 & 30,71 & 44,27 & 9,079 & 60,48 \\
\hline \multirow[t]{12}{*}{ Mt. Garnet } & 171 & 22,47 & 26,82 & 0,452 & 0,735 & 0,302 & 29,96 & 45,39 & 8,749 & 61,95 \\
\hline & 172 & 22,48 & 27,11 & 0,453 & 0,722 & 0,302 & 31,44 & 47,36 & 8,743 & 59,78 \\
\hline & 173 & 22,52 & 27,66 & 0,455 & 0,717 & 0,304 & 31,15 & 46,57 & 9,105 & 59,92 \\
\hline & 174 & 22,55 & 27,51 & 0,455 & 0,710 & 0,304 & 31,38 & 45,60 & 8,840 & 59,79 \\
\hline & 175 & 22,50 & 26,57 & 0,452 & 0,735 & 0,302 & 31,72 & 45,81 & 8,965 & 59,20 \\
\hline & 176 & 22,46 & 27,06 & 0,452 & 0,707 & 0,301 & 31,93 & 45,28 & 8,890 & 58,95 \\
\hline & 177 & 22,56 & 28,42 & 0,457 & 0,727 & 0,305 & 31,93 & 46,13 & 8,847 & 58,98 \\
\hline & 178 & 22,50 & 27,72 & 0,454 & 0,720 & 0,303 & 30,62 & 45,89 & 8,073 & 61,53 \\
\hline & 179 & 22,52 & 27,18 & 0,453 & 0,702 & 0,302 & 31,23 & 46,03 & 8,601 & 60,20 \\
\hline & 180 & 22,49 & 27,77 & 0,455 & 0,716 & 0,304 & 31,65 & 44,93 & 8,230 & 59,88 \\
\hline & 181 & 22,54 & 28,57 & 0,457 & 0,714 & 0,305 & 30,83 & 44,71 & 7,737 & 61,49 \\
\hline & 182 & 22,48 & 26,54 & 0,452 & 0,694 & 0,301 & 31,34 & 44,52 & 7,968 & 60,56 \\
\hline Médias & & 22,51 & 27,51 & 0,454 & 0,719 & 0,303 & 31,24 & 45,80 & 8,63 & 60,11 \\
\hline Desvio $\mathrm{Pa}$ & adrão & 0,034 & 0,627 & 0,002 & 0,010 & 0,001 & 0,521 & 1,132 & 0,446 & 0,887 \\
\hline
\end{tabular}

DAP - Diâmetro à Altura do Peito $(\mathrm{cm})$, ALTT - altura total $(\mathrm{m})$; VCC - volume comercial com casca $\left(\mathrm{m}^{3}\right.$ árvore-1); DBM densidade básica da madeira $\left(\mathrm{g} \mathrm{cm}^{-3}\right)$, BIOMT - biomassa do tronco (kg árvore $\left.{ }^{-1}\right), \mathrm{LIG}, \mathrm{CEL}, \mathrm{EXT}$ e HOLO respectivamente, teores (\%) de lignina, celulose, extrativos e holocelulose. 
As progênies foram agrupadas em três grupos pelo método de agrupamento de Ward considerando o valor genotípico estimado (Figura 1). Os grupos 1 e 2 englobam progênies de maiores DAP, ALTT, VCC e BIOMT, portanto mais produtivas e com pequenas diferenças entre ambos, e o grupo 3 as de menor produtividade (Tabela 3). Quanto aos caracteres de qualidade da madeira, o grupo 2 apresenta maiores teores de LIG e EXT e menores teores de HOLO; o grupo 1 tem maiores teores de CEL e de HOLO e os menores de LIG e EXT e o grupo 3 os menores teores de CEL (Tabela 3). Portanto, os grupos 1 e 2 diferenciam-se, principalmente, quanto às características da madeira, os grupos 1 e 3, diferenciam-se pela produtividade, e os grupos 2 e 3 diferenciam-se tanto pela produtividade quanto pelas características da madeira.

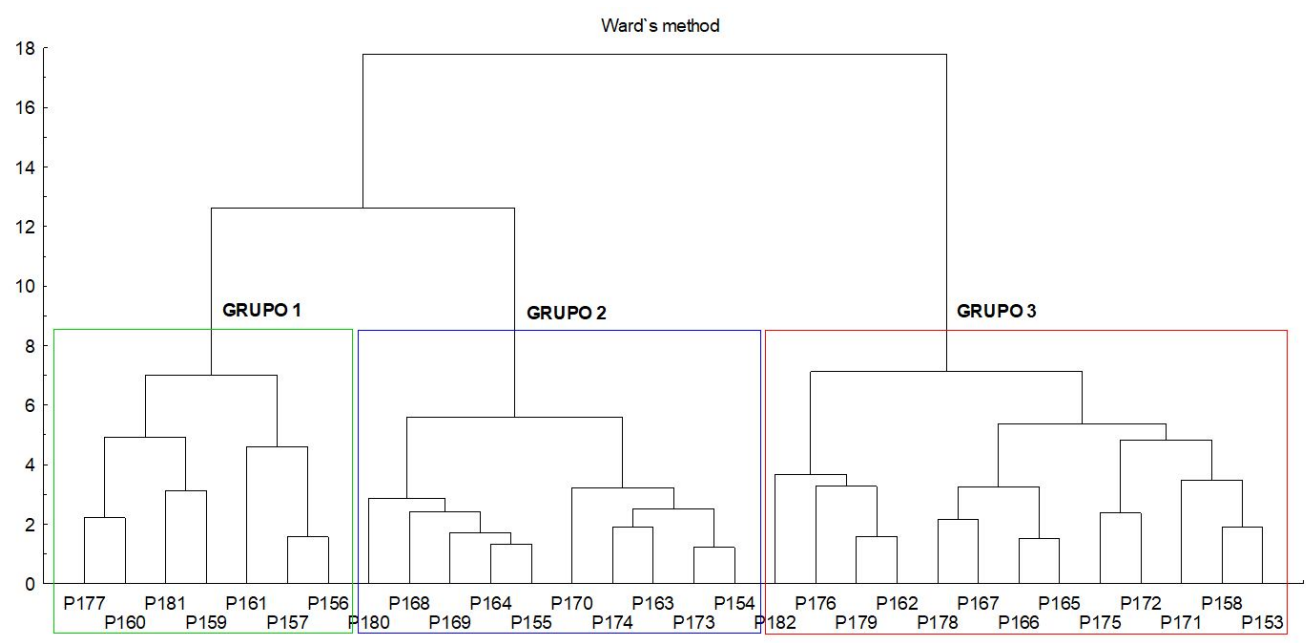

Figura 1. Dendrograma baseado na Distância Euclidiana entre 30 progênies de polinização aberta de Eucalyptus tereticornis pertencentes a duas procedências Australianas, obtido pelo método Ward a partir de nove caracteres de crescimento e de qualidade da madeira.

Tabela 3. Médias genotípicas dos caracteres de crescimento e de qualidade da madeira, dos grupos formados pelo método de Ward, em 30 progênies de polinização aberta de Eucalyptus tereticornis pertencentes a duas procedências Australianas.

\begin{tabular}{cccc}
\hline & \multicolumn{3}{c}{ Médias } \\
\hline Características & Grupo 1 & Grupo 2 & Grupo 3 \\
DAP & 22,55 & 22,53 & 22,48 \\
ALTT & 28,44 & 27,57 & 27,04 \\
VCC & 0,457 & 0,455 & 0,453 \\
DBM & 0,724 & 0,715 & 0,718 \\
BIOMT & 0,305 & 0,304 & 0,302 \\
LIG & 31,11 & 31,53 & 31,06 \\
CEL & 46,83 & 45,34 & 45,56 \\
EXT & 8,248 & 8,881 & 8,667 \\
HOLO & 60,59 & 59,47 & 60,35
\end{tabular}

DAP - Diâmetro à Altura do Peito $(\mathrm{cm})$, ALTT - altura total $(\mathrm{m})$; VCC - volume comercial com casca $\left(\mathrm{m}^{3}\right.$ árvore $\left.{ }^{-1}\right)$; DBM densidade básica da madeira $\left(\mathrm{g} \mathrm{cm}^{-3}\right)$, BIOMT - biomassa do tronco ( $\mathrm{kg}$ árvore-1), LIG, CEL, EXT e HOLO respectivamente, teores (\%) de lignina, celulose, extrativos e holocelulose.

No agrupamento formado pelo método não-hierárquico K-means (Figura 2), cujo número de grupos (3) foi pré-definido a partir dos resultados obtidos no agrupamento de Ward, a composição dos grupos foi pouco alterada em relação àqueles formados no método de Ward. Observa-se que no grupo 1 do método de K-means, 6 das 7 progênies que o constitui pertencem ao grupo 1 de Ward e representa progênies de maior crescimento e produtividade, maior DBM, CEL e HOLO e menores valores de LIG e EXT. 
No grupo 2 de K-means, 10 das 11 progênies que o compõe pertencem ao grupo 2 de Ward, sendo representado por progênies com produtividade intermediária, porém com maiores teores de LIG e EXT e menores valores de DBM, CEL e HOLO.

O grupo 3 no método de $K$-means, formado por 12 progênies, apresenta 11 progênies pertencentes ao grupo 3 de Ward apresentando progênies de menor produtividade e valores intermediários para DBM, LIG, CEL, EXT e HOLO. Percebe-se, também, por esse método que os três grupos reúnem progênies das duas procedências, reforçando o pequeno efeito de procedências para a separação e composição dos grupos.

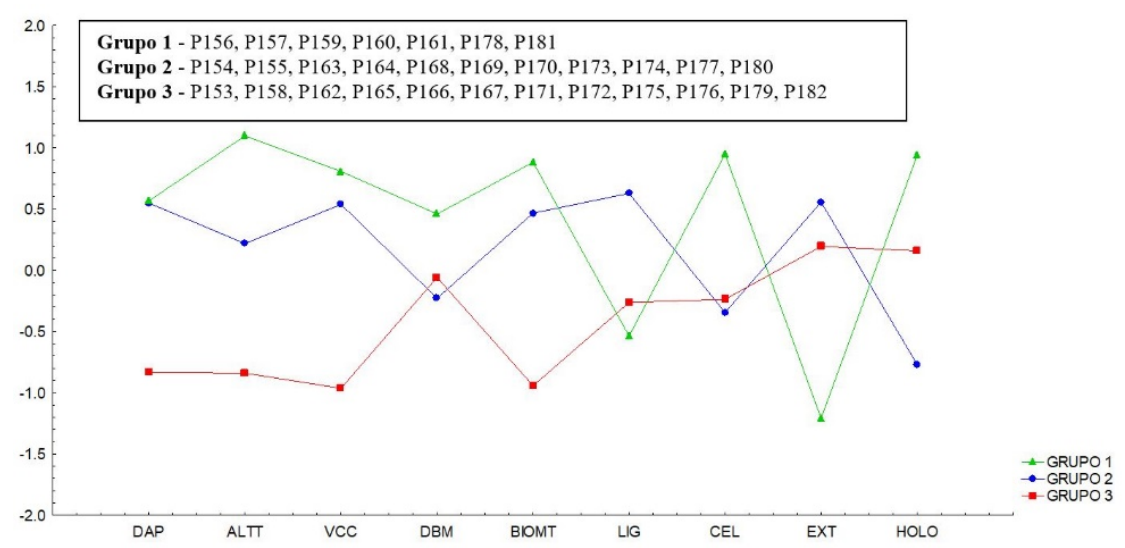

Figura 2. Caracterização dos grupos pelo método não-hierárquico K-means, formados a partir da avaliação de nove caracteres de crescimento e de qualidade da madeira em 30 progênies de polinização aberta de Eucalyptus tereticornis pertencentes a duas procedências Australianas.

Observa-se, assim, que apesar da relativa semelhança entre os grupos formados nos métodos de Ward (Figura 1; Tabela 3) e de K-means (Figura 2), que as pequenas alterações na composição dos mesmos possibilitaram uma melhor definição dos grupos, principalmente, quanto à produtividade. Isto confere maior confiabilidade na identificação de progênies de comportamentos semelhantes e, ou contrastantes.

Quanto ao padrão de dispersão das progênies a partir da análise de componentes principais (CP), os três primeiros CPs explicam 78,49\% da variância contida nas variáveis originais (Tabela 4) e estão dentro do critério de Kaiser (1961), que considera autovalores maiores que 1. Assumindo como relevantes os caracteres cujas cargas apresentam valor absoluto superior a 0,50 (Tobar-Tosse et al., 2015), os caracteres de crescimento (DAP, ALTT, VCC e BIOMT) apresentam maior poder discriminatório no componente principal 1 (CP1). Os caracteres com maior poder discriminatório no CP2 são os teores de HOLO, LIG e EXT, sendo que o teor de HOLO, por apresentar sinal positivo, atua de forma inversa aos outros dois que apresentam sinais negativos. No CP3 foram relevantes os caracteres DBM e teor de CEL, ambos atuando no mesmo sentido.

Os caracteres em destaque nos três componentes principais têm grande importância para seleção das progênies mais promissoras para um programa de melhoramento. As características mais correlacionadas com o CP1 são aquelas associadas ao crescimento vegetal, portanto, este é o componente relacionado com a produtividade das progênies. As progênies de maior crescimento e produtividade localizam-se na parte direita das Figuras 3 e 4 . As características mais correlacionadas com CP2 são associadas aos constituintes da madeira, mas com comportamentos distintos, notadamente, quanto aos teores de LIG e HOLO; assim, as progênies localizadas na parte inferior da Figura 3 têm maiores teores de LIG e de EXT em contraposição àquelas situadas na parte superior dessa figura, que apresentam maiores teores de HOLO. O CP3 está associado com os teores de CEL e com a DBM.

$\mathrm{O}$ ângulo formado entre os vetores dos caracteres de crescimento (fixando o caráter VCC para melhor compreensão e visualização; eixo " $x$ " - CP1) e os vetores dos caracteres de qualidade da madeira (eixo "y" - CP2 e CP3) é de aproximadamente 90 graus, o que significa baixa correlação. De fato, são encontradas uma quase-independência entre as naturezas desses dois grupos de 
caracteres (Hamilton et al., 2009; Stackpole et al., 2011; Denis et al., 2013). Isso é um fator interessante para as estratégias de melhoramento, uma vez que permite a seleção de árvores produtivas sem comprometer a qualidade da madeira. No entanto é necessária cautela nas tomadas de decisões, pois, as correlações entre crescimento e caracteres de qualidade da madeira podem variar com a população e local (Chen et al., 2018).

Tabela 4. Correlações fator-variáveis dos caracteres de crescimento e de qualidade da madeira nos três primeiros componentes principais para 30 progênies de polinização aberta de Eucalyptus tereticornis pertencentes a duas procedências Australianas.

\begin{tabular}{cccc}
\hline & CP1 & CP2 & CP3 \\
\hline DAP & 0.7803 & -0.2548 & 0.3024 \\
ALTT & 0.9426 & 0.0597 & -0.0322 \\
VCC & 0.9714 & -0.1085 & 0.1269 \\
DBM & 0.1692 & 0.3282 & -0.7778 \\
BIOMT & 0.9771 & -0.0302 & -0.0330 \\
LIG & 0.0625 & -0.8493 & -0.2894 \\
CEL & 0.2769 & 0.3167 & -0.6310 \\
EXT & -0.2613 & -0.5548 & -0.0590 \\
HOLO & 0.0443 & 0.9570 & 0.2804 \\
Autovalores & 3,5752 & 2,2342 & 1,2785 \\
Variância (\%) & 39,7252 & 24,8244 & 14,2066 \\
Variância Acumulada (\%) & 39,7253 & 64,5497 & 78,7564 \\
\hline
\end{tabular}

DAP - Diâmetro à Altura do Peito $(\mathrm{cm})$, ALTT - altura total $(\mathrm{m})$; VCC - volume comercial com casca $\left(\mathrm{m}^{3}\right.$ árvore $\left.{ }^{-1}\right)$; DBM densidade básica da madeira $\left(\mathrm{g} \mathrm{cm}^{-3}\right)$, BIOMT - biomassa do tronco ( $\mathrm{kg}$ árvore-1), LIG, CEL, EXT e HOLO respectivamente, teores (\%) de lignina, celulose, extrativos e holocelulose.

Entre as progênies mais produtivas (à direita de CP1 - Figuras 3 e 4), P159, P181, P161 e P157 apresentam baixos teores de LIG e EXT, enquanto a P177 possui teores elevados (eixo " $y$ " - CP2; Figura 3). Essas substâncias são elimidadas pelas indústrias de cellulose porque reduzem o rendimento da polpa no processo fabril (Fantuzzi Neto, 2012; Denis et al., 2013). Nesse contexto, a progênie P159 é a mais promissora, pois possui os maiores teores de CEL e alta DBM dentre as citadas (eixo " $\mathrm{y}$ " - CP3; Figura 4). A progênie P158, apesar de estar entre as menos produtivas, também, encontra-se entre as de baixos teores de LIG e EXT e altos de CEL, além de elevada DBM, portanto, não sendo descartado seu uso num programa de melhoramento para melhoria na qualidade da madeira. De outro modo, a progênie P182 é a menos indicada para seleção por ser pouco produtiva, baixo teor de CEL e baixa DBM. As progênies dos grupos 1 (cor verde - Figuras 3 e 4) e 2 (cor azul - Figuras 3 e 4) de Ward, divergem quanto a alguns constituintes da qualidade da madeira, notadamente quanto aos teores de LIG e de HOLO. Assim, dependendo do objetivo do programa de melhoramento pode-se priorizar progênies distintas pelas características da madeira, embora com produtividade semelhante.

Nas Figuras 3 e 4, as progênies localizadas dentro da elipse apresentam comportamentos próximos, sem grandes diferenças entre si, sendo, portanto, mais generalistas. Aquelas que se localizam fora das elipses se destacam por alguma(s) característica(s) específica(s) e devem, portanto, merecer atenção especial nos programas de melhoramento.

O uso de diferentes métodos de análise multivariada objetivou a comparação dos resultados de forma a permitir uma identificação mais precisa das progênies de desempenho similares e divergentes. Percebe-se que, independentemente do método de agrupamento, de maneira geral, os caracteres de crescimento influenciaram mais na separação dos grupos que os caracteres de qualidade da madeira (Figuras 1 a 3; Tabela 2), mas estas permitiram a discriminação de progênies de produtividade semelhante, porém com propriedades químicas distintas.

O número de grupos formados em estudos de divergência genética depende de vários fatores, desde aqueles relacionados à técnica de formação dos grupos, como a medida de distância e o método de agrupamento, até ao germoplasma considerado em termos de 
número de acessos, além da variabilidade genética e o número e tipo de caracteres avaliados. A princípio, o pequeno número de grupos identificado indica a presença de baixa variabilidade genética nas progênies em estudo.

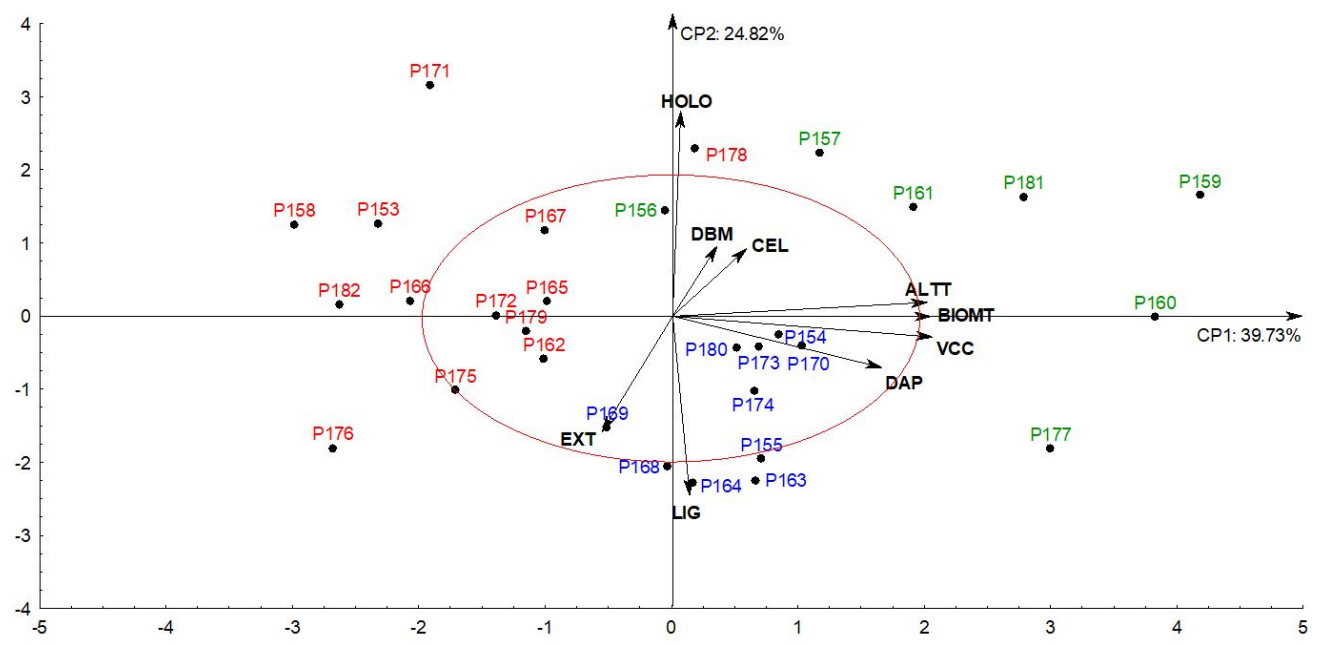

Figura 3. Gráfico biplot com a dispersão de 30 progênies de polinização aberta de Eucalyptus tereticornis pertencentes a duas procedências para as nove caracteres avaliados nos componentes principais 1 e 2 (CP1/CP2). As cores verde, azul e vermelho representam respectivamente os grupos de progênies $1,2 \mathrm{e}$

3 formados pelo método de Ward. HOLO: holocelulose; DBM: densidade básica da madeira; CEL: celulose; ALTT: altura total; BIOMT: biomassa total; VCC: volume com casca; DAP: diâmetro da altura do peito; LIG: lignina; EXT: extrativos.

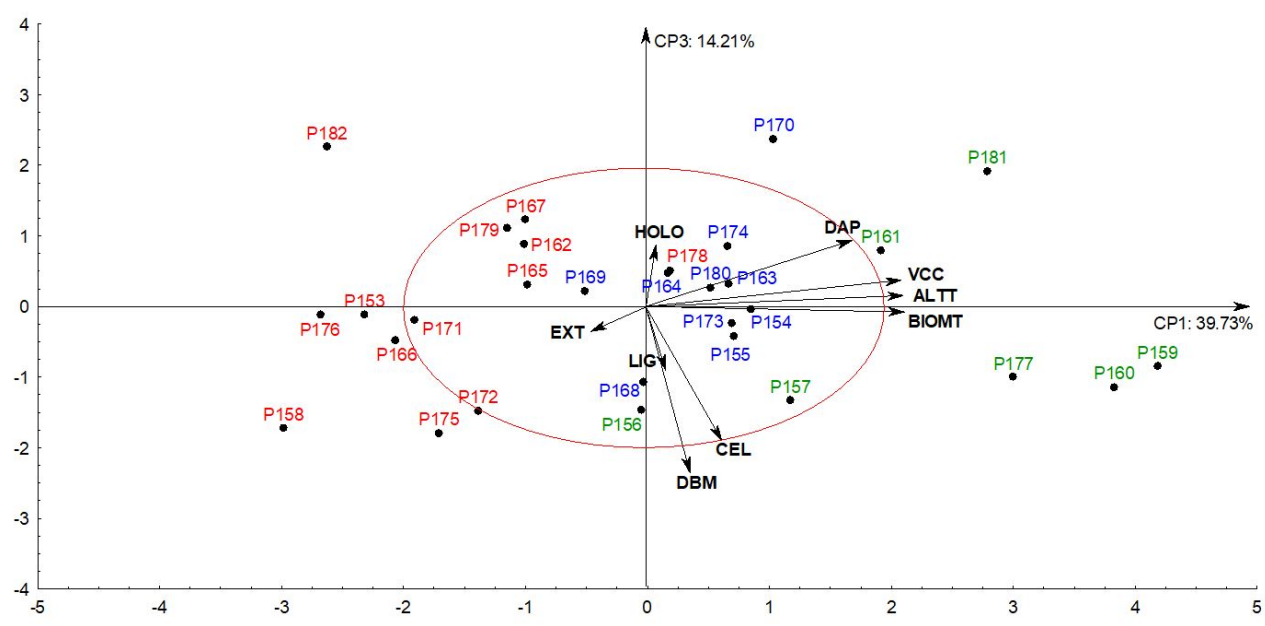

Figura 4. Gráfico biplot com a dispersão de 30 progênies polinização aberta de Eucalyptus tereticornis pertencentes as duas procedências para as nove características avaliadas nos componentes principais 1 e 3 (CP1/CP3). As cores verde, azul e vermelho representam respectivamente os grupos de progênies 1, 2 e 3 formados pelo método de Ward. HOLO: holocelulose; DBM: densidade básica da madeira; CEL: celulose; ALTT: altura total; BIOMT: biomassa total; VCC: volume com casca; DAP: diâmetro da altura do peito; LIG: lignina; EXT: extrativos.

\section{CONCLUSÃO}

A divergência genética entre as 30 progênies de polinização aberta de Eucalyptus tereticornis não está associada à procedência das progênies estudadas. As progênies avaliadas diferem quanto ao potencial produtivo e caracteres de qualidade da madeira. Os grupos de progênies identificados podem ser utilizados nas estratégias de melhoramento de modo a selecionar progênies superiores com finalidade de ganho genético em produtividade e 
melhoria na qualidade da madeira para segmentos distintos, de acordo com o objetivo do programa de melhoramento.

\section{AGRADECIMENTOS}

O presente trabalho foi realizado com apoio da Coordenação de Aperfeiçoamento de Pessoal de Nível Superior - Brasil (CAPES) - Código de Financiamento 001. Ao CNPq pela bolsa de produtividade em pesquisa ao segundo e quarto autores. Ao Instituto Florestal, em especial ao PqC Antonio Carlos Scatena Zanatto.

\section{REFERÊNCIAS}

Alves, R. M., Garcia, A. A. F., Cruz, E. D., \& Figueira, A. (2003). Seleção de descritores botânicoagronômicos para a caracterização de germoplasma de cupuaçuzeiro. Pesquisa Agropecuária Brasileira, 38(7), 807-818. http://dx.doi.org/10.1590/S0100-204X2003000700004.

Balbinot, E., Carneiro, J. G. A., Baroso, D. G., \& Paes, H. M. F. (2010). Crescimento inicial de Eucalyptus tereticornis em plantios puro e consorciado com Mimosa caesalpiniifolia e Mimosa pilulifera, em Campos dos Goytacazes - RJ. Revista Árvore, 34(1), 1-11. http://dx.doi.org/10.1590/S010067622010000100001.

Brooker, M. H. I., \& Kleinig, D. A. (2006). Field guide to Eucalypts (3rd ed., Vol. 1). Melbourne: Boomings Books.

Chen, S., Weng, Q., Li, F., Li, M., Zhou, C., \& Gan, S. (2018). Genetic parameters for growth and wood chemical properties in Eucalyptus urophylla $\times$ E. tereticornis hybrids. Annals of Forest Science, 75(1), 16. http://dx.doi.org/10.1007/s13595-018-0694-x.

Climate-Date. (2018). Recuperado em 12 de outubro de 2018, de https://pt.climate-data.org/

Cornelius, J. (1994). Heritabilities and additive genetic coefficients of variation in forest trees. Canadian Journal of Forest Research, 24(2), 372-379. http://dx.doi.org/10.1139/x94-050.

Denis, M., Favreau, B., Ueno, S., Camus-Kulandaivelu, L., Chaix, G., Gion, J.-M., Nourrisier-Mountou, S., Polidori, J., \& Bouvet, J.-M. (2013). Genetic variation of wood chemical traits and association with underlying genes in Eucalyptus urophylla. Tree Genetics \& Genomes, 9(4), 927-942. http://dx.doi.org/10.1007/s11295-013-0606-z.

Eldridge, K., Davidson, J., Harwood, C., \& Van Wyk, G. (1993). Eucalypt domestication and breeding. New York: Oxford University Press.

Fantuzzi Neto, H. (2012). Qualidade da madeira de eucalipto para produção de celulose kraft (Tese de doutorado). Universidade Federal de Viçosa, Viçosa.

Fernandes, E. F., Gomide, J. L., Colodette, J. L., \& Ferreira, M. Z. (2011). Influência da produtividade de clones híbridos de eucalipto na densidade da madeira e na polpação Kraft. Scientia Forestalis, 39(90), 143-150.

Flores, T. B., Alvares, C. A., Souza, V. C., \& Stape, J. L. (2016). Eucalyptus no Brasil: zoneamento climático e guia para identificação. Piracicaba: IPEF.

Foelkel, C. E. B., Mora, E., \& Menochelli, S. (1992). Densidade básica: sua verdadeira utilidade como índice de qualidade da madeira de eucalipto para produção de celulose. O Papel, (5). Recuperado em 7 de outubro de 2018, de http://www.celsofoelkel.com.br/artigos/outros/Db\%20sua\%20verdadeira\%20utilidade.pdf

Fonseca, S. M., Resende, M. D. V., Alfenas, A. C., Guimarães, L. M. S., Assis, T. F., \& Grattapaglia, D. (2010). Manual prático de melhoramento genético do eucalipto. Viçosa: UFV.

Garcia, C. H. (1989). Tabelas para classificação do coeficiente de variação (Circular Técnica IPEF, No. 171، 12 p.). Piracicaba: IPEF.

Gomide, J. L., \& Colodette, J. L. (2007). Qualidade da madeira. In A. Borém (Ed.), Biotecnologia florestal (pp. 25-54). Viçosa: UFV.

Gomide, J. L., \& Demuner, B. J. (1986). Determinação do teor de lignina em material lenhoso: método Klason modificado. O Papel, 47(8), 36-38.

Gonçalves, J. L. M., Alvares, C. A., Rocha, J. H. T., Brandani, C. B., \& Hakamada, R. (2017). Eucalypt plantation management in regions with water stress (pp. 1-15). Grahamstown: Southern Forests. 
Hamilton, M. G., Raymond, C. A., Harwood, C. E., \& Potts, B. M. (2009). Genetic variation in Eucalyptus nitens pulpwood and wood shrinkage traits. Tree Genetics \& Genomes, 5(2), 307-316. http://dx.doi.org/10.1007/s11295-008-0179-4.

He, X., Li, F., Li, M., Weng, Q., Shi, J., Mo, X., \& Gan, S. (2012). Quantitative genetics of cold hardiness and growth in Eucalyptus as estimated from E. urophylla $\times$ E. tereticornis hybrids. New Forests, 43(3), 383394. http://dx.doi.org/10.1007/s11056-011-9287-3.

Indústria Brasileira de Árvores - IBÁ. (2017). Relatório 2017. São Paulo. Recuperado em 15 de agosto de 2018, de http://iba.org/images/shared/Biblioteca/IBA_RelatorioAnual2017.pdf

Indústria Brasileira de Árvores - IBÁ. (2019). Relatório 2019. São Paulo. Recuperado em 15 de outubro de 2019, de https://www.iba.org/datafiles/publicacoes/relatorios/iba-relatorioanual2019.pdf

Instituto de Pesquisas e Estudos Florestais - IPEF. (2018) Chave de identificação de espécies florestais (CIEF): Eucalyptus terenticornis SM. Piracicaba. Recuperado em 18 de junnho de 2018, de http://www.ipef.br/identificacao/cief/especies/terenticornis.asp

Kaiser, H. F. (1961). A note on guttman's lower bound for the number of common factors. British Journal of Statistical Psychology, 14(1), 1-2. http://dx.doi.org/10.1111/j.2044-8317.1961.tb00061.x.

Mojena, R. (1977). Hierarchical grouping methods and stopping rules: an evaluation. The Computer Journal, 20(4), 359-363. http://dx.doi.org/10.1093/comjnl/20.4.359.

Morais, S. A. L., Nascimento, E. A., \& Melo, D. C. (2005). Análise da madeira de Pinus oocarpa. Parte I Estudos dos constituintes macromoleculares e extrativos voláteis. Revista Árvore, 29(3), 461-470. http://dx.doi.org/10.1590/S0100-67622005000300014.

Pimentel Gomes, F. (1990). Curso de estatística experimental (12. ed.). São Paulo: Nobel.

Resende, M. D. V. (2007). Selegen-Reml/Blup: sistema estatístico e seleção genética computadorizada via modelos lineares mistos. Colombo: Embrapa Florestas.

Resende, M. D. V., Mora, A. L., Higa, A. R., \& Paludzyszyn Filho, E. (1998). Efeito do tamanho amostral na estimativa da herdabilidade em espécies perenes. Floresta, 28(1-2), 51-63.

Rowell, R. M., Pettersen, R., Han, J. S., Rowell, J. S., \& Tshabalala, M. A. (2005). Cell wall chemistry. In R. M. Rowell (Ed.), Handbook of wood chemistry and wood composites (Chap. 3). New York: Taylor \& Francis Group. http://dx.doi.org/10.1201/9780203492437.

Santos, C. R., \& Sansígolo, C. A. (2000). Métodos não-normatizados para determinação de celulose como parâmetro de seleção de árvores matrizes visando a produção de polpa Kraft-AQ. In Ibero American Congress on Pulp and Paper (13 p.).

Santos, H. G., Jacomine, P. K. T., Anjos, L. H. C., Oliveira, V. A., Lumbreras, J. F., Coelho, M. R., Almeida, J. A., Cunha, T. J. F., \& Oliveira, J. B. (2013). Sistema brasileiro de classificação de solos (3. ed. rev. e ampl.). Brasília: Embrapa.

Silva, F. F., Silva, D. D., \& Arnold, A. (2007). Avaliação do desempenho inicial de procedências de Eucalyptus tereticornis Smith no Vale do Rio Doce-MG. Ciência Agronômica, 38(3), 270-275.

Silva, J. C., Matos, J. L. M., Oliveira, J. T. S., \& Evangelista, W. V. (2005). Influência da idade e da posição ao longo do tronco na composição química da madeira de Eucalyptus grandis Hill ex. Maiden. Revista Árvore, 29(3), 455-460. http://dx.doi.org/10.1590/S0100-67622005000300013.

Soares, C. P. B., Paula Neto, F., \& Souza, A. L. (2006). Dendrometria e inventário florestal. Viçosa: UFV.

Soares, V. C., Bianchi, M. L., Trugilho, P. F., Hofler, J., \& Pereira, A. J. (2015). Análise das propriedades da madeira e do carvão vegetal de híbridos de eucalipto em três idades. Cerne, 21(2), 191-197. http://dx.doi.org/10.1590/01047760201521021294.

Stackpole, D. J., Vaillancourt, R. E., Alves, A., Rodrigues, J., \& Potts, B. M. (2011). Genetic variation in the chemical components of Eucalyptus globulus wood. G3: Genes, Genomes, Genetics, 1(2), 151-159. PMid:22384327. http://dx.doi.org/10.1534/g3.111.000372.

StatSoft. (2004). Statistica: data analysis software system. Version 7. Recuperado em 12 de outubto de 2018, de http://www.statsoft.com

Tobar-Tosse, D. E., Castoldi, R., Candido, W. S., Ferraudo, A. S., Charlo, H. C. O., \& Braz, L. T. (2015). Caracterização de genótipos de soja hortaliça por análise de componentes principais. Ciência Rural, 45(7), 1214-1219. http://dx.doi.org/10.1590/0103-8478cr20140597.

Trugilho, P. F., Lima, J. T., \& Mendes, L. M. (1996). Influência da idade nas características físicoquímicas e anatômicas da madeira de Eucalyptus saligna. Cerne, 2(1), 94-116.

Vital, B. R. (1984). Métodos de determinação de densidade da madeira (Boletim Técnico, No. 1, 21 p.). Viçosa: SIF. 
Vital, B. R., Carneiro, A. C. O., \& Pereira, B. L. C. (2013). Qualidade da madeira para fins energéticos. In F. Santos, J. L. Colodette \&J. H. Queiroz (Eds.), Bioenergia e biorrefinaria: cana-de-açúcar e espécies florestais (pp. 321-354). Viçosa: UFV.

Volker, P. W., Potts, B. M., \& Borralho, N. M. G. (2008). Genetic of intra-and inter-specific hybrids of Eucalyptus globulus and E. nitens. Tree Genetics \& Genomes, 4(3), 445-460. http://dx.doi.org/10.1007/s11295-007-0122-0.

Williams, E. R., Matheson, A. C., \& Harwood, C. E. (2002). Experimental design and analysis for tree improvement (2nd e.). Collingwood: CSIRO Publishing. http://dx.doi.org/10.1071/9780643090132.

Contribuição dos autores: BZ - conceituação, curadoria de dados, investigação, metodologia, escrita - primeira redação, escrita - revisão e edição; RCP -conceituação, curadoria de dados, análise formal, investigação, metodologia, administração do projeto; escrita - primeira redação, escrita - revisão e edição; NFP -conceituação, metodologia, escrita - primeira redação, escrita - revisão e edição; MLMF - conceituação, metodologia, escrita primeira redação; MJA - curadoria de dados, metodologia, escrita - primeira redação, escrita - revisão e edição. 